\title{
Sensorless Exact Input-Output Linearization Control of the Induction Machine, Based on Parallel Stator Resistance and Speed MRAS Observer, with a Flux Sliding Mode Observer
}

\author{
Mohamed Moutchou and Hassan Mahmoudi \\ Mohamed V University Agdal, Rabat \\ Mohammadia School's of Engineer, LEEP, Morocco
}

\begin{abstract}
This paper presents an exact input-output linearization control scheme for rotor speed and rotor flux control of induction motor drive. In this scheme the motor model, is described in the fixed stator frame, and it is linearized, using exact inputoutput linearization technique, allowing a decoupled control of rotor flux and torque. To avoid the use of mechanical sensor, the rotor speed estimation is made by an observer using a specific MRAS (Model reference adaptive system) technique; this observer is designed to perform simultaneous estimation of stator resistance and rotor speed. In order to estimate rotor flux, a sliding mode observer is proposed in this paper. Simulation results are realized and presented to validate and to prove the effectiveness of the proposed Sensorless control.
\end{abstract}

Keywords: Input-output linearization, Induction Motor Drive, MRAS observer, Sliding Mode.

\section{Introduction}

Induction motors are widely used in industry especially in variable speed applications. An induction motor is simple in operation, rugged, maintenance free and generally less expensive than other machines.

However, its model is complicated for various reasons. The dynamic behavior of the motor is described by a fifth-order highly coupled and nonlinear dynamical system, the rotor electric variables (fluxes and currents) are practically not measured; and some of its physical parameters may vary significantly while operating the motor (stator and mainly rotor resistance, due to heating, magnetizing induction due to saturation );

Those difficulties have whetted the curiosity of scientists and researcher in laboratories, in the last few years, Evidenced by the growing number of publications that discuss the subject. Different control strategies were developed, like Field Oriented Control (FOC) proposed by Blaschke [1], and Direct Torque Control (DTC) have been extensively reported and discussed in the literature [1]-[4] to achieve a decoupled control of induction motor.

In vector control the torque and flux are decoupled by a suitable decoupling network. Then the flux component and the torque are controlled independently and respectively by stator direct-axis current and stator quadratic-axis current to control the induction motor (IM) as a separately excited DC motor.

A disadvantage of the FOC is that the method assumes that the magnitude of the rotor flux is regulated to a constant value. Therefore, the rotor speed is only asymptotically decoupled from the rotor flux. To improve the I.M control performances others technique were conceived like the sliding mode control, it is characterized by simplicity of design and attractive robustness properties. Its major drawback is the chattering phenomenon [5]-[8].

By contrast, the passivity based control $[9,10]$, doesn't cancel all the nonlinearity but ensure system stability, but its experimental implementation is still difficult.

Backstepping control approach [11]-[15], is more recent. Its present form is due to Krstic, Kanellakopoulos and Kokotovic. This control technique offers good performance in both steady state and transient operations.

Received: August $23^{\text {rd }}, 2013$. Accepted: May $7^{\text {th }}, 2014$ 
Among technique that we find in the literature, there is the exact input-output linearization technique [16]-[23] used in this work. The technique of input-output linearization based on the differential geometry allows by a diffeomorphic transformation and a state feedback to uncouple and linearize the model put under canonical and then controlled using linear control techniques. This technique has the advantage of being able to separately control flux and torque even in mode of variation of flux. This method cancels the nonlinear terms in the plant model which fails when the physical parameters varies.

These control techniques can not guarantee good performances without the use of suitable state observer. Among the observation technique used, there are the sliding mode techniques used in this work to estimate flux, and MRAS technique, used, in particular, in Sensorless IM drives at the first time by Schauder (1992). MRAS is interesting since it leads to relatively easy to implement system with high speed adaptation [24]-[27].

In this work we propose a new structure of MRAS Observer, allowing simultaneous observation of stator resistance and rotor speed.

This article is organized into three main sections, in the first one we design the exact inputoutput linearization control, in the second one we present the flux sliding mode observer, the last one we present the design of the parallel MRAS observer of stator resistance and rotor speed.

Simulation results, given at the end, illustrate the good performance of this combination of control method and observation techniques.

\section{Input-Output Linearization of the Induction Machine}

In order to reduce the complexity of the three phase model and then simplify the control design, an equivalent two phase representation is chosen. Under the assumptions of linearity of the magnetic circuit and neglecting iron losses, a two phase IM model in the fixed stator, reference frame $(\alpha, \beta)$ can be described as:

$$
\left\{\begin{array}{l}
\frac{d \Phi_{r \alpha}}{d t}=\lambda_{r} \cdot\left(L_{m} \cdot i_{s \alpha}-\Phi_{r \alpha}\right)-p \cdot \Omega \cdot \Phi_{r \beta} \\
\frac{d \Phi_{r \beta}}{d t}=\lambda_{r} \cdot\left(L_{m} \cdot i_{s \beta}-\Phi_{r \beta}\right)+p \cdot \Omega \cdot \Phi_{r \alpha} \\
\frac{d i_{s \alpha}}{d t}=-\gamma \cdot i_{s \alpha}+k \cdot \lambda_{r} \cdot \Phi_{r \alpha}+k \cdot p \cdot \Omega \cdot \Phi_{r \beta}+\delta \cdot v_{s \alpha} \\
\frac{d i_{s \beta}}{d t}=-\gamma \cdot i_{s \beta}-k \cdot p \cdot \Omega \cdot \Phi_{r \alpha}+k \cdot \lambda_{r} \cdot \Phi_{r \beta}+\delta \cdot v_{s \beta} \\
\frac{d \Omega}{d t}=\frac{\mu}{J} \cdot\left(\Phi_{r \alpha} \cdot i_{s \beta}-\Phi_{r \beta} \cdot i_{s \alpha}\right)-\frac{f}{J} \cdot \Omega-\frac{T_{L}}{J}
\end{array}\right.
$$

Where:

$$
\begin{aligned}
& \sigma=1-\frac{L_{m}{ }^{2}}{L_{r} \cdot L_{s}} ; \quad k=\frac{L_{m}}{\sigma L_{s} L_{r}} ; \quad T_{r}=\frac{L_{r}}{R_{r}} ; \quad \lambda_{r}=\frac{R_{r}}{L_{r}} ; \gamma=\frac{1}{\sigma L_{s}}\left(R_{s}+\frac{R_{r} \cdot L_{m}{ }^{2}}{L_{r}{ }^{2}}\right) ; \quad \mu=p \frac{L_{m}}{L_{r}} ; \\
& \delta=\frac{1}{\sigma \cdot L_{s}}
\end{aligned}
$$

We saw that the dynamic equations of the induction machine are non-linear, what make the control difficult to conceive. However, with some transformations, the nonlinear system can be converted into the corresponding linear system. Feedback linearization is one of the approaches for the nonlinear control design [18], [19]. The fundamental idea is to apply linear control techniques for the nonlinear system. It has been used to solve a lot of practical control 
problems in industry by transforming a nonlinear system dynamics into a fully or partly linear one. The simplest form of feedback linearization is to cancel the nonlinearities of a nonlinear system so that the closed-loop dynamics is in a linear form.

The structure of the proposed I.M control is represented below, in Figure 1.

The induction motor model (1) is put in the following form:

$$
\left\{\begin{array}{l}
\dot{x}=f(x)+g(x) \\
y=h(x)
\end{array}\right.
$$

Where:

$$
\begin{aligned}
& x=\left[\Phi_{r \alpha}, \Phi_{r \beta}, i_{s \alpha}, i_{s \beta}, \Omega\right]^{T} \\
& f(x)=\left[\begin{array}{l}
-\lambda_{r} \cdot \Phi_{r \alpha}-p \cdot \Omega_{r \beta}+\lambda_{r} \cdot L_{m} \cdot i_{s \alpha} \\
p \cdot \Omega \cdot \Phi_{r \alpha}-\lambda_{r} \cdot \Phi_{r \beta}+\lambda_{r} \cdot L_{m} \cdot i_{s \beta} \\
k \cdot \lambda_{r} \cdot \Phi_{r \alpha}+k \cdot p \cdot \Omega \cdot \Phi_{r \beta}-\gamma \cdot i_{s \alpha} \\
-k \cdot p \cdot \Omega_{r \alpha}+k \cdot \lambda_{r} \cdot \Phi_{r \beta}-\gamma \cdot i_{s \beta} \\
\frac{\mu}{J} \cdot\left(\Phi_{r \alpha} \cdot i_{s \beta}-\Phi_{r \beta} \cdot i_{s \alpha}\right)-\frac{f}{J} \cdot \Omega-\frac{T_{L}}{J}
\end{array}\right] \\
& g(x)=\left[\begin{array}{ccccc}
0 & 0 & 0 & \delta . u_{\alpha} & 0 \\
0 & 0 & \delta . u_{\alpha} & 0 & 0
\end{array}\right]^{T}
\end{aligned}
$$

Having two control variables $u_{\alpha}$ and $u_{\beta}$, it is possible to decompose the model into two independent systems and then control separately two outputs. We choose as the outputs, the electromagnetic torque Te and the rotor flux modulus $\Phi_{r}{ }^{2}$.

$$
h(x)=\left[\begin{array}{c}
T_{e} \\
\Phi_{r}{ }^{2}
\end{array}\right]=\left[\begin{array}{c}
\mu \cdot\left(\Phi_{r \alpha} \cdot i_{s \beta}-\Phi_{r \beta} \cdot i_{s \alpha}\right) \\
\Phi_{r \alpha}{ }^{2}+\Phi_{r \beta}{ }^{2}
\end{array}\right]
$$

In order to be able to impose arbitrary dynamics on every output $y_{1}=T_{e}$ and $y_{2}=\Phi_{r}{ }^{2}$, we mast find a differential relation linear between the output $y_{1}$ and $y_{2}$ and input of command $u_{\alpha}$ and $u_{\beta}$, it is necessary to find a return of state, in a way that the system in closed buckle is linear and decoupled. Thus, it is necessary to derive the output function $h_{1}(x)$ and $h_{2}(x)$ respectively $r_{1}$ and $r_{2}$ (corresponding relative degrees) time, until create differential equations where intervene the commands $\left(u_{\alpha}, u_{\beta}\right)$.

This linearization operation is possible in case where the total relative degree $r=r_{1}+r_{2}$ is lower or equal to the order of the system $n(r \leq n)$, so the system is controllable. By successive derivation we can write:

$$
\left\{\begin{array}{l}
\frac{d y_{1}^{r_{1}}}{d t}=L_{f}{ }^{r_{1}} \cdot h_{1}(x)+\sum_{j=1}^{2} L_{g_{j}} \cdot L_{f}^{r_{1}-1} \cdot h_{1}(x) \cdot u_{j} \\
\frac{d y_{2}{ }^{r_{2}}}{d t}=L_{f}^{r_{2}} \cdot h_{2}(x)+\sum_{j=1}^{2} L_{g_{j}} \cdot L_{f}^{r_{2}-1} \cdot h_{2}(x) \cdot u_{j}
\end{array}\right.
$$


$L_{f} h$ is $h$ lie derivation in direction of the vector field $f_{(\text {.) }}$ such as:

$$
\left\{\begin{array}{l}
L_{f} h(x)=d h . f=\sum_{i}^{n} \frac{\partial h(x)}{\partial x_{i}} \cdot f_{i}(x) \\
f(x)=\left(f_{1}(x), f_{2}(x), \ldots . . f_{n}(x)\right)
\end{array}\right.
$$

And $r=\left[r_{1}, r_{2}\right]$ is the relative degree which has to satisfy the following conditions:

$$
\begin{array}{ll}
* L_{g_{j}} L_{f}{ }^{k} \cdot h_{i}(x)=0 & \text { for }\left\{\begin{array}{l}
1 \leq j \leq 2, \\
1 \leq i \leq 2, k<r_{i}-1,
\end{array}\right. \\
* L_{g_{j}} L_{f}{ }^{r_{i}-1} \cdot h_{i}(x) \neq 0 \quad \text { for } \quad & \left\{\begin{array}{l}
1 \leq j \leq 2, \\
1 \leq i \leq 2,
\end{array}\right.
\end{array}
$$

Also let us define the matrix of decoupling:

$$
D(x)=\left[\begin{array}{cc}
L_{g_{1}} L_{f}^{r_{1}-1} \cdot h_{1}(x) & L_{g_{2}} L_{f}^{r_{1}-1} \cdot h_{1}(x) \\
L_{g_{1}} L_{f}^{r_{2}-1} \cdot h_{2}(x) & L_{g_{2}} L_{f}^{r_{2}-1} \cdot h_{2}(x)
\end{array}\right]
$$

We can then write the system of equation (3) under the following matrix form:

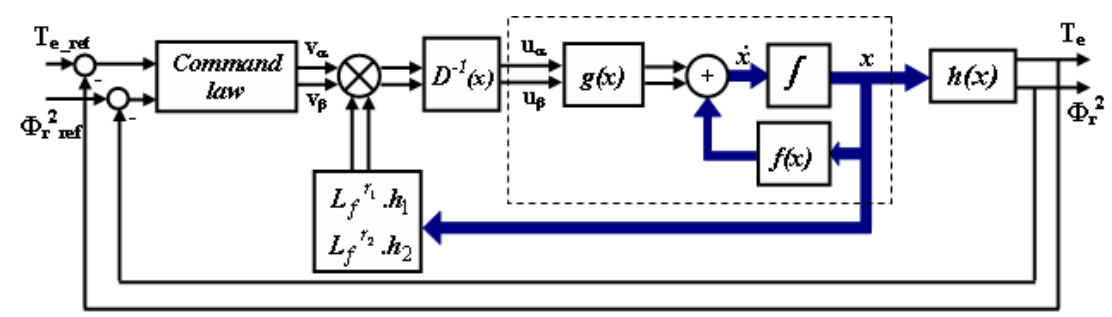

Figure 1. Structure of the nonlinear control (input-output linearization)

$$
\left[\begin{array}{l}
h_{1}^{\left(r_{1}\right)}(x) \\
h_{2}{ }^{\left(r_{2}\right)}(x)
\end{array}\right]=\left[\begin{array}{c}
L_{f}^{r_{1}} \cdot h_{1} \\
L_{f}{ }^{r_{2}} \cdot h_{2}
\end{array}\right]+D(x) \cdot\left[\begin{array}{l}
u_{\alpha} \\
u_{\beta}
\end{array}\right]
$$

\section{A. For the first output: torque Te}

Consider the machine model defined by (2) and determine the order of derivative for which the first variable to be set (the electromagnetic torque) is explicitly affected by the controls $u_{\alpha}$ and $u_{\beta}$.

By successive derivations, the relative degree $r_{1}$ associated with the torque of the MAS is equal 1:

$$
\begin{aligned}
& \frac{d y_{1}}{d t}=\frac{d T_{e}}{d t}=L_{f} h_{1}(x)+L_{g_{1}} L_{f}^{0} h_{1}(x) \cdot u_{\alpha}+L_{g_{2}} L^{0}{ }_{f} h_{1}(x) \cdot u_{\beta} \\
& L_{f} h_{1}(x)=\mu \cdot\left(\Phi_{r \alpha}^{\prime} \cdot i_{s \beta}+\Phi_{r \alpha} \cdot i_{s \beta}^{\prime}-\Phi_{r \beta}^{\prime} \cdot i_{s \alpha}-\Phi_{r \beta} \cdot i_{s \alpha}^{\prime}\right)
\end{aligned}
$$

Then we get this expression:

$$
\begin{aligned}
L_{f} h_{1}(x)= & A_{1} \cdot\left(\Phi_{r \alpha} \cdot i_{s \beta}-\Phi_{r \beta} \cdot i_{s \alpha}\right)+A_{2} \cdot\left(\Phi_{r \alpha} \cdot i_{s \alpha}+\Phi_{r \beta} \cdot i_{s \beta}\right) \\
& +A_{3} \cdot\left(\Phi_{r \alpha}{ }^{2}+\Phi_{r \beta}{ }^{2}\right)
\end{aligned}
$$


Where:

$$
\left\{\begin{array}{l}
A_{1}=-\mu \cdot\left(\lambda_{r}+\gamma\right) \\
A_{2}=-p \cdot \Omega \cdot \mu \\
A_{3}=k \cdot A_{2}
\end{array}\right.
$$

And:

$$
\left\{\begin{array}{l}
L_{g_{1}} L_{f}^{0} h_{1}(x)=-p \cdot k \cdot \Phi_{r \beta} \\
L_{g_{2}} L^{0}{ }_{f} h_{1}(x)=p \cdot k \cdot \Phi_{r \alpha}
\end{array}\right.
$$

B. For the second output: square of rotor flux $\Phi_{r}{ }^{2}$.

In the same way as previously, the relative degree $r_{2}$, associated with the second output, is equal 2. Then after the second derivation we get:

$$
\frac{d^{2} y_{2}}{d t^{2}}=L_{f}^{2} h_{2}(x)+L_{g_{1}} L^{1} h_{2}(x) \cdot u_{\alpha}+L_{g_{2}} L^{1} h_{2}(x) \cdot u_{\beta}
$$

Where:

$$
\begin{gathered}
L_{f} h_{2}(x)=-2 \cdot \lambda_{r} \cdot\left(\Phi_{r \alpha}{ }^{2}+\Phi_{r \beta}{ }^{2}\right)+2 \cdot L_{m} \cdot \lambda_{r} \cdot\left(\Phi_{r \alpha} \cdot i_{s \alpha}+\Phi_{r \beta} \cdot i_{s \beta}\right) \\
\Rightarrow L^{2}{ }_{f} h_{2}(x)=B_{1} \cdot\left(i_{s \alpha}{ }^{2}+i_{s \beta}{ }^{2}\right)+B_{2} \cdot\left(\Phi_{r \alpha} \cdot i_{s \beta}-\Phi_{r \beta} \cdot i_{s \alpha}\right)+ \\
B_{3} \cdot\left(\Phi_{r \alpha} \cdot i_{s \alpha}+\Phi_{r \beta} \cdot i_{s \beta}\right)+B_{4} \cdot\left(\Phi_{r \alpha}{ }^{2}+\Phi_{r \beta}{ }^{2}\right)
\end{gathered}
$$

Where:

$$
\begin{aligned}
& \left\{\begin{array}{lll}
B_{1}=2 \cdot\left(L_{m} \cdot \lambda_{r}\right)^{2} & , & B_{2}=2 \cdot p \cdot \Omega \cdot L_{m} \cdot \lambda_{r} \\
B_{3}=-6 \cdot L_{m} \cdot \lambda_{r}{ }^{2}-2 \cdot \gamma \cdot L_{m} \cdot \lambda_{r} & , & B_{4}=4 \cdot \lambda_{r}{ }^{2}+2 \cdot k \cdot L_{m} \cdot \lambda_{r}{ }^{2}
\end{array}\right. \\
& \left\{\begin{array}{l}
L_{g_{1}} L_{f}^{1} h_{2}(x)=2 \cdot k \cdot R_{r} \cdot \Phi_{r \alpha} \\
L_{g_{2}} L_{f}{ }_{f} h_{2}(x)=2 . k \cdot R_{r} \cdot \Phi_{r \beta}
\end{array}\right.
\end{aligned}
$$

\section{Feedback law}

The matrix form of the system equations (4) allows as deducting the return of nonlinear state $u=\alpha(x)+\beta(x) \cdot v$ that give to the system a linear input/output behavior:

By putting:

$$
\left\{\begin{array}{l}
\alpha(x)=-D^{-1}(x) \cdot\left[\begin{array}{c}
L_{f}^{{ }_{1}} \cdot h_{1} \\
L_{f}^{r_{2}} \cdot h_{2}
\end{array}\right] \\
\beta(x)=D^{-1}(x)
\end{array}\right.
$$

The input of the resulting linear system is defined as follow:

$$
\cdot\left[\begin{array}{l}
u_{\alpha} \\
u_{\beta}
\end{array}\right]=D^{-1}(x)\left(\left[\begin{array}{c}
v_{\alpha} \\
v_{\beta}
\end{array}\right]-\left[\begin{array}{c}
L_{f}^{r_{1}} . h_{1} \\
L_{f}^{r_{2}} . h_{2}
\end{array}\right]\right)
$$

Where:

$$
v_{\alpha} \text { and } v_{\beta}: \text { are the new inputs controls. }
$$




$$
\begin{aligned}
D(x) & =\left[\begin{array}{ll}
L_{g_{1}} L_{f}^{0} \cdot h_{1}(x) & L_{g_{2}} L_{f}^{0} \cdot h_{1}(x) \\
L_{g_{1}} L_{f}^{1} \cdot h_{2}(x) & L_{g_{2}} L_{f}^{1} \cdot h_{2}(x)
\end{array}\right] \\
& =\left[\begin{array}{cc}
-p \cdot k \cdot \Phi_{r \beta} & p \cdot k \cdot \Phi_{r \alpha} \\
2 . k \cdot R_{r} \cdot \Phi_{r \alpha} & 2 . k \cdot R_{r} \cdot \Phi_{r \beta}
\end{array}\right]
\end{aligned}
$$

The commands $u_{\alpha}$ and $u_{\beta}$ can be determined if the decoupling matrix $\mathrm{D}(\mathrm{x})$ is not singular, thus $\mathrm{D}(\mathrm{x})$ is invertible if $\Phi_{r \alpha}{ }^{2}+\Phi_{r \beta}{ }^{2} \neq 0$.

Care must be taken to this singularity condition in the simulation as well as implementation of the control strategy. At the starting point of the simulation/implementation, the flux magnitude is zero. In case of digital implementation of the system, to avoid numerical saturation of the processor, this singularity can be avoided by putting a small positive value for the flux magnitude.

By applying this feedback law (5), a new dynamic model of the machine is obtained, which is represented by the both differential equations below:

$$
\left[\begin{array}{l}
h_{1}{ }^{\left(r_{1}\right)}(x) \\
h_{2}{ }^{\left(r_{2}\right)}(x)
\end{array}\right]=\left[\begin{array}{c}
\frac{d T_{e}}{d t} \\
\frac{d^{2}\left(\Phi_{r}{ }^{2}\right)}{d t^{2}}
\end{array}\right]=\left[\begin{array}{c}
v_{\alpha} \\
v_{\beta}
\end{array}\right]
$$

\section{Control law}

Now, we define the control law, which allows automatic control of electromagnetic torque $T_{e}$ to $T_{e_{r e f}}$ and rotor flux modulus $\Phi_{r}{ }^{2}$ to $\Phi_{r e f}{ }^{2}$, as follow:

$$
\left\{\begin{array}{l}
v_{\alpha}=-k_{1} \cdot\left(T_{e}-T_{e_{r e f}}\right)+\dot{T}_{e_{r e f}} \\
v_{\beta}=-k_{2} \cdot\left(\Phi^{2}{ }_{r}-\Phi^{2}{ }_{r e f}\right)-k_{3} \cdot\left(\dot{\Phi}^{2}{ }_{r}-\dot{\Phi}^{2}{ }_{r e f}\right)+\ddot{\Phi}^{2}{ }_{r e f}
\end{array}\right.
$$

We define the variables errors as:

$$
\left\{\begin{array}{l}
e_{1}=\left(T_{e}-T_{e_{r e f}}\right) \\
e_{2}=\left(\Phi^{2}{ }_{r}-\Phi^{2}{ }_{r e f}\right)
\end{array}\right.
$$

So the dynamics of errors is determined by:

$$
\left\{\begin{array}{l}
\dot{e}_{1}+k_{1} \cdot e_{1}=0 \\
\ddot{e}_{2}+k_{3} \cdot \dot{e}_{2}+k_{2} \cdot e_{2}=0
\end{array}\right.
$$

The dynamics of the two errors will be stable if the roots of polynomials corresponding characteristics are placed in the left half-plane of the complex plane.

\section{E. Speed loop PI corrector Determination}

Speed loop is the outer loop and the torque is the inner loop of the first decoupled subsystem, where $h_{1}(x)=T_{e}$ is the output and $v_{\alpha}$ is the input. So the torque loop must be faster than the speed. In this case the block diagram representing this structure is as follows: 


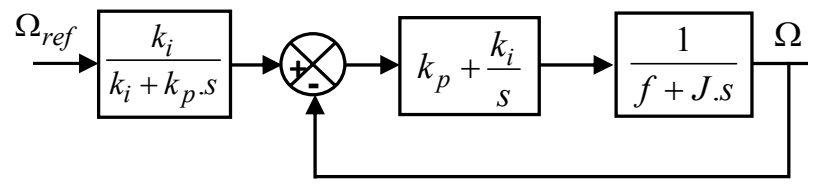

Figure 2. Block-scheme of the speed loop below:

According to this scheme, we can determine the transfer function of the speed loop as

$$
\frac{\Omega(s)}{\Omega_{r e f}(s)}=\frac{1}{1+\left(\frac{k_{p}+f}{k_{i}}\right) \cdot s+\left(\frac{J}{k_{i}}\right) \cdot s^{2}}
$$

and then calculate the suitable P.I parameters $\left(k_{i}, k_{p}\right)$.

\section{Rotor Flux Sliding Mode Observer (SMO)}

\section{A. Induction machine model}

In this section we use a model of asynchronous machine formulated in a stationary stator reference frame $(\alpha, \beta)$, as showing below:

$$
\left\{\begin{array}{l}
\dot{I}_{s}=-\gamma \cdot I_{s}+k \cdot A \cdot \Phi_{r}+\delta \cdot V_{s} \\
\dot{\Phi}_{r}=L_{m} \cdot \lambda_{r} \cdot I_{s}-A \cdot \Phi_{r}
\end{array}\right.
$$

With:

$$
\begin{aligned}
& I_{s}=\left[\begin{array}{ll}
i_{s \alpha} & i_{s \beta}
\end{array}\right]^{T} ; \\
& \Phi_{r}=\left[\begin{array}{ll}
\Phi_{r \alpha} & \Phi_{r \beta}
\end{array}\right]^{T} \\
& A=\left[\begin{array}{cc}
\lambda_{r} & \omega \\
-\omega & \lambda_{r}
\end{array}\right] ; \\
& V_{s}=\left[\begin{array}{ll}
v_{s \alpha} & v_{s \beta}
\end{array}\right]^{T}
\end{aligned}
$$

\section{B. Rotor flux SMO model}

The equations representing the observer model are given below:

$$
\left\{\begin{array}{l}
\dot{\hat{I}}_{s}=-\gamma \cdot \hat{I}_{s}+k \cdot A \cdot \hat{\Phi}_{r}+\delta \cdot V_{s}+D_{i} \cdot u_{s} \\
\dot{\hat{\Phi}}_{r}=L_{m} \cdot \lambda_{r} \cdot \hat{I}_{s}-A \cdot \hat{\Phi}_{r}+D_{\varphi} \cdot u_{s}
\end{array}\right.
$$

The dynamics of the estimation error is expressed by the following equations:

$$
\left\{\begin{array}{l}
\dot{\tilde{I}}_{s}=-\gamma \cdot \widetilde{I}_{s}+k \cdot A \cdot \widetilde{\Phi}_{r}-D_{i} \cdot u_{s} \\
\dot{\widetilde{\Phi}}_{r}=L_{m} \cdot \lambda_{r} \cdot \widetilde{I}_{s}-A \cdot \widetilde{\Phi}_{r}-D_{\varphi} \cdot u_{s}
\end{array}\right.
$$

Where:

- $\widetilde{I}_{s}=I_{s}-\hat{I}_{s}:$ is the stator current estimation error.

- $\widetilde{\Phi}_{r}=\Phi_{r}-\hat{\Phi}_{r}$ : is the rotor flux estimation error.

- $u_{s}=\left[\operatorname{sign}\left(S_{1}\right) \operatorname{sign}\left(S_{2}\right)\right]^{T}$

- $S=\left[s_{1} s_{2}\right]^{T}=\eta \cdot\left(\widetilde{I}_{S}\right):$ is the sliding mode surface.

- $D_{\varphi}, D_{i}, \eta$ : are the matrix $(2 \times 2)$ that we will determine later. 
C. Design of the rotor flux SMO

We consider the following Lyapunov candidate function:

$V=\frac{1}{2} \cdot S^{T} \cdot S$

Its time derivative is the following:

$$
\dot{V}=S^{T} \dot{S}
$$

We suppose that $\frac{d \eta}{d t}=0$, thus we obtain:

$$
\begin{aligned}
& \dot{V}=S^{T} \cdot \eta \cdot \dot{\widetilde{I}}_{s} \\
& \dot{V}=S^{T} \cdot \eta \cdot\left(-\gamma \cdot \widetilde{I}_{s}+k \cdot A \cdot \widetilde{\Phi}_{r}\right)-S^{T} \cdot \eta \cdot D_{i} \cdot u_{s}
\end{aligned}
$$

In order to have $\dot{V}$ negative definite and satisfy the condition of attractiveness, we must have:

$$
S^{T} \cdot \eta \cdot\left(-\gamma \cdot \widetilde{I}_{s}+k \cdot A \cdot \widetilde{\Phi}_{r}\right)<S^{T} \cdot \eta \cdot D_{i} \cdot u_{s}
$$

If we put:

$$
\eta \cdot D_{i}=\left[\begin{array}{ll}
\mu_{1} & 0 \\
0 & \mu_{2}
\end{array}\right]
$$

Then we obtain the condition below:

$$
\mu_{1} \cdot\left|S_{1}\right|+\mu_{2} \cdot\left|S_{2}\right|>S^{T} . \eta \cdot\left(-\gamma \cdot \widetilde{I}_{S}+k \cdot A \cdot \widetilde{\Phi}_{r}\right)
$$

When the sliding mode will be reached, the switching surface will verify:

$$
\widetilde{I}_{s}=\dot{\widetilde{I}}_{s}=0
$$

Therefore we obtain:

$$
\Rightarrow u_{s}=D_{i}^{-1} \cdot k \cdot A \cdot \widetilde{\Phi}_{r}
$$

By putting this equation in (18) we get this equation:

$$
\dot{\widetilde{\Phi}}_{r}=-\left(A+D_{\varphi} \cdot D_{i}^{-1} \cdot k \cdot A\right) \widetilde{\Phi}_{r}
$$

We put:

$$
\begin{aligned}
& A+D_{\varphi} \cdot D_{i}^{-1} \cdot k \cdot A=\mathrm{P} \\
& \Rightarrow \dot{\widetilde{\Phi}}_{r}=-\mathrm{P} \cdot \widetilde{\Phi}_{r}
\end{aligned}
$$

In order to have an exponential convergence we choose $\mathrm{P}$ under the following form:

$$
\mathrm{P}=\left[\begin{array}{ll}
p_{1} & 0 \\
0 & p_{2}
\end{array}\right]
$$

Where $\mathrm{p} 1$ and $\mathrm{p} 2$ are a positives constants.

Then we obtain de following equation:

$$
D_{\varphi}=(\mathrm{P}-A) \cdot A^{-1} \cdot k^{-1} \cdot D_{i}
$$


Now if we put:

$$
\eta=A^{-1} \cdot k^{-1}
$$

So we finally find the following equations:

$$
D_{i}=k \cdot A \cdot\left[\begin{array}{ll}
\mu_{1} & 0 \\
0 & \mu_{2}
\end{array}\right] \quad ; \quad D_{\varphi}=(\mathrm{P}-A) \cdot\left[\begin{array}{ll}
\mu_{1} & 0 \\
0 & \mu_{2}
\end{array}\right]
$$

Finally the condition of attractiveness becomes:

$$
\mu_{1} \cdot\left|S_{1}\right|+\mu_{2} \cdot\left|S_{2}\right|>S^{T} \cdot \tilde{\Phi}_{r}
$$

To complete the design of the observer, it now suffices to choose properly the observer parameters. The $\left(p_{1}, p_{2}\right)$ parameters must be chosen to determine the dynamics of observer convergence and the $\left(\mu_{1}, \mu_{2}\right)$ parameters must be chosen to satisfy the condition of attractiveness and stability of the observer.

\section{Parallel Stator Resistance and Rotor Speed MRAS Observer}

The simultaneous estimation of rotor speed and the stator resistance is realized in this section by using a technique based on a MRAS structure composed of three models and two adaptation mechanisms providing the estimate of the rotor speed and stator resistance.

The structure of this observer is given in Figureure 3, it shows that stator resistance is estimated using a voltage model adjustable by it, which provided an estimate of the stator current. The stator current estimation error will be used by an adaptive mechanism to estimate the value of stator resistance.

The speed estimation is performed in parallel with that of stator resistance; it is based on an adaptation mechanism that uses the error between the estimated flux components supplied by a current model, adjustable by rotor speed estimation, and the flux components provided by the flux sliding mode observer.

The following Figure represents the observation technique structure.

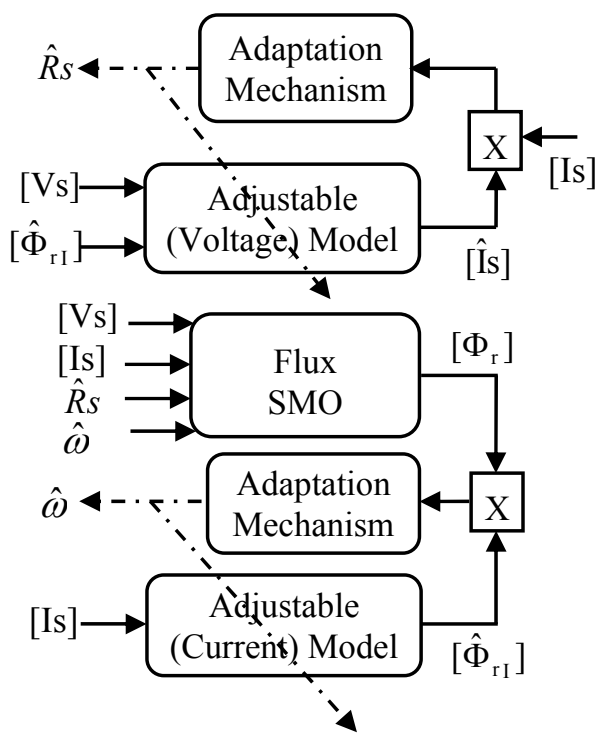

Figure 3. Parallel MRAS Observer structure 
By using the dynamic model of the asynchronous machine, formulated in a stator reference frame $(\alpha, \beta)$, and by using measurements of the stator currents and voltages, we build two estimators one for stator currents estimation the others for rotor flux estimation.

\section{A. Adjustable Current Model}

Consider the two first equation of induction machine model (1):

$$
\left\{\begin{array}{l}
\frac{d \hat{\Phi}_{r \alpha I}}{d t}=\lambda_{r} \cdot\left(L_{m} \cdot i_{s \alpha}-\hat{\Phi}_{r \alpha I}\right)-\hat{\omega} \cdot \hat{\Phi}_{r \beta I} \\
\frac{d \hat{\Phi}_{r \beta I}}{d t}=\lambda_{r} \cdot\left(L_{m} \cdot i_{s \beta}-\hat{\Phi}_{r \beta I}\right)+\hat{\omega} \cdot \hat{\Phi}_{r \alpha I}
\end{array}\right.
$$

This model is adjustable by rotor speed, it provide the flux estimation by using measured stator current and estimated rotor speed.

\section{B. Adjustable Voltage Model}

We can use the following voltage model as adjustable model for stator resistance estimation:

$$
\left\{\begin{array}{l}
\frac{d \hat{i}_{s \alpha}}{d t}=\delta \cdot\left[v_{s \alpha}-L_{m} \cdot \lambda_{r} \cdot \frac{d \hat{\Phi}_{r \alpha I}}{d t}-\hat{R}_{s} \cdot \hat{i}_{s \alpha}\right] \\
\frac{d \hat{i}_{s \beta}}{d t}=\delta \cdot\left[v_{s \beta}-L_{m} \cdot \lambda_{r} \cdot \frac{d \hat{\Phi}_{r \beta I}}{d t}-\hat{R}_{s} \cdot \hat{i}_{s \beta}\right]
\end{array}\right.
$$

In this model we use the measured voltage and the estimated flux provided by the adjustable current model, to estimate stator current.

We define the following estimation errors, respectively of speed, stator resistance, rotor flux and stator current:

$$
\begin{aligned}
& \int \widetilde{\omega}=\omega-\hat{\omega} \\
& \left\{\widetilde{R}_{s}=R_{s}-\hat{R}_{s}\right. \\
& \left\{\begin{array}{l}
\widetilde{\Phi}_{r \alpha}=\Phi_{r \alpha}-\hat{\Phi}_{r \alpha I} \\
\widetilde{\Phi}_{r \beta}=\Phi_{r \beta}-\hat{\Phi}_{r \beta I}
\end{array}\right. \\
& \left\{\begin{array}{l}
\tilde{i}_{s \alpha}=i_{s \alpha}-\hat{i}_{s \alpha} \\
\tilde{i}_{s \beta}=i_{s \beta}-\hat{i}_{s \beta}
\end{array}\right.
\end{aligned}
$$

After deriving errors, we obtain the errors dynamic equations as follow:

$$
\left\{\begin{array}{l}
\dot{\widetilde{\Phi}}_{r \alpha}=-\lambda_{r} \cdot \tilde{\Phi}_{r \alpha}-\omega \cdot \Phi_{r \beta}+\hat{\omega} \cdot \hat{\Phi}_{r \beta I} \\
\dot{\widetilde{\Phi}}_{r \beta}=-\lambda_{r} \cdot \tilde{\Phi}_{r \beta}+\omega \cdot \Phi_{r \alpha}-\hat{\omega} \cdot \hat{\Phi}_{r \alpha I} \\
\dot{\vec{i}}_{s \alpha}=-\delta \cdot\left(R_{s} \cdot i_{s \alpha}-\hat{R}_{s} \hat{i}_{s \alpha}\right) \\
\dot{\vec{i}}_{s \beta}=-\delta \cdot\left(R_{s} \cdot i_{s \beta}-\hat{R}_{s} \hat{i}_{s \beta}\right)
\end{array}\right.
$$


By considering relation (11, 12 and 13), we can rewrite (14) as below:

$$
\left\{\begin{array}{l}
\dot{\widetilde{\Phi}}_{r \alpha}=-\lambda_{r} \cdot \widetilde{\Phi}_{r \alpha}-\widetilde{\omega} \cdot \Phi_{r \beta}-\hat{\omega} \cdot \widetilde{\Phi}_{r \beta} \\
\dot{\tilde{\Phi}}_{r \beta}=-\lambda_{r} \cdot \widetilde{\Phi}_{r \beta}+\widetilde{\omega} \cdot \Phi_{r \alpha}+\hat{\omega} \cdot \widetilde{\Phi}_{r \alpha} \\
\dot{\widetilde{i}}_{s \alpha}=-\delta \cdot\left(\hat{R}_{s} \cdot \tilde{i}_{s \alpha}+\widetilde{R}_{s} \cdot i_{s \alpha}\right) \\
\dot{\widetilde{i}}_{s \beta}=-\delta .\left(\hat{R}_{s} \cdot \tilde{i}_{s \beta}+\widetilde{R}_{s} \cdot i_{s \beta}\right)
\end{array}\right.
$$

C. Stability Analysis

In order to determine the observer stability condition, and then determine the adaptation mechanism that gives us the speed estimation, let us consider the following LCF:

$$
V=\frac{1}{2} \cdot \tilde{i}_{s \alpha}^{2}+\frac{1}{2} \cdot \tilde{i}_{s \beta}{ }^{2}+\frac{\widetilde{R}_{s}^{2}}{2}+\frac{1}{2} \cdot \widetilde{\Phi}_{r \alpha}{ }^{2}+\frac{1}{2} \cdot \widetilde{\Phi}_{r \beta}{ }^{2}+\frac{\widetilde{\omega}^{2}}{2}
$$

The LCF derivative is as below:

$$
\begin{aligned}
\dot{V}= & -\delta \cdot \hat{R}_{s} \cdot\left(\widetilde{i}_{s \alpha}{ }^{2}+\widetilde{i}_{s \beta}{ }^{2}\right)-\delta \cdot\left(i_{s \alpha} \cdot \widetilde{i}_{s \alpha}+i_{s \beta} \cdot \widetilde{i}_{s \beta}\right) \cdot \widetilde{R}_{s}+\dot{\widetilde{R}}_{s} \cdot \widetilde{R}_{s} \\
& -\lambda_{r} \cdot\left(\widetilde{\Phi}_{r \alpha}{ }^{2}+\widetilde{\Phi}_{r \beta}{ }^{2}\right)+\left(\Phi_{r \alpha} \cdot \widetilde{\Phi}_{r \beta}-\Phi_{r \beta} \cdot \widetilde{\Phi}_{r \alpha}\right) \cdot \widetilde{\omega}+\dot{\tilde{\omega}} \cdot \widetilde{\omega} \\
\dot{V} & \leq \widetilde{R}_{s} \cdot\left[-\delta \cdot\left(i_{s \alpha} \cdot \widetilde{i}_{s \alpha}+i_{s \beta} \cdot \widetilde{i}_{s \beta}\right)-\dot{\hat{R}}\right]+\widetilde{\omega} \cdot\left[\Phi_{r \alpha} \cdot \widetilde{\Phi}_{r \beta}-\Phi_{r \beta} \cdot \widetilde{\Phi}_{r \alpha}-\dot{\hat{\omega}}\right]
\end{aligned}
$$

In order to make $\dot{V}$ to be negative definite, we can for example force the second term to be null, then we can write:

$$
\left\{\begin{array}{l}
\dot{\hat{R}}=-\delta \cdot\left(i_{s \alpha} \cdot \tilde{i}_{s \alpha}+i_{s \beta} \cdot \tilde{i}_{s \beta}\right) \\
\dot{\hat{\omega}}=\left(\Phi_{r \alpha} \cdot \widetilde{\Phi}_{r \beta}-\Phi_{r \beta} \cdot \widetilde{\Phi}_{r \alpha}\right)
\end{array}\right.
$$

We have:

$$
\dot{\widetilde{\omega}}=-\dot{\hat{\omega}} \text { and } \dot{\widetilde{R}}_{s}=-\dot{\hat{R}}_{s}
$$

Then the corresponding adaptive law, that ensures the stability of the MRAS observer, is as follow:

$$
\begin{aligned}
& \hat{\omega}=\int\left(\Phi_{r \alpha} \cdot \widetilde{\Phi}_{r \beta}-\Phi_{r \beta} \cdot \widetilde{\Phi}_{r \alpha}\right) \cdot d t \\
& \hat{R}=-\int \delta \cdot\left(i_{s \alpha} \cdot \tilde{i}_{s \alpha}+i_{s \beta} \cdot \tilde{i}_{s \beta}\right) \cdot d t
\end{aligned}
$$

In order to decrease response temp of estimation and ensure a null steady error, we use PI controller as follow:

$$
\begin{aligned}
& \hat{\omega}=k p_{1}\left(\Phi_{r \alpha} \cdot \widetilde{\Phi}_{r \beta}-\Phi_{r \beta} \cdot \widetilde{\Phi}_{r \alpha}\right)+k i_{1} \cdot \int\left(\Phi_{r \alpha} \cdot \widetilde{\Phi}_{r \beta}-\Phi_{r \beta} \cdot \widetilde{\Phi}_{r \alpha}\right) \cdot d t \\
& \hat{R}=-k p_{2} \cdot\left(i_{s \alpha} \cdot \tilde{i}_{s \alpha}+i_{s \beta} \cdot \tilde{i}_{s \beta}\right)-k i_{2} \cdot \int\left(i_{s \alpha} \cdot \tilde{i}_{s \alpha}+i_{s \beta} \cdot \tilde{i}_{s \beta}\right) \cdot d t
\end{aligned}
$$

Where $\left(k p_{1}, k p_{2}\right)$ and $\left(k i_{1}, k i_{2}\right)$ are the proportional and integral positive gain, choosing to have good dynamic performance of the observer.

The last variable we need to know is the electromagnetic torque, which we choose to estimate using the following expression:

$\hat{T}_{e}=\mu \cdot\left(\hat{\Phi}_{r \alpha} \cdot i_{s \beta}-\hat{\Phi}_{r \beta} \cdot i_{s \alpha}\right)$ 


\section{Simulation Results}

Using Matlab/Simulink, the proposed controller technique and observer performances have been verified by the following simulation results.

In order to ensure that the references are differentiable, and then the control law (5) to be realizable, in these simulations the references steps of speed and Flux are filtered, by a secondorder low-pass filter with cut-off pulsation equal to $500 \mathrm{rd} / \mathrm{s}$.

The Figure 4 shows us the simulation responses of the system commanded for a speed reference steps Figure 4a, in order to allows the observer to achieve the actual value of stator resistance, the first step is given at $0.5 \mathrm{~s}$ from 0 to $100 \mathrm{rad} / \mathrm{s}$, the second one is from $100 \mathrm{rad} / \mathrm{s}$ to $150 \mathrm{rad} / \mathrm{s}$ at $1.5 \mathrm{~s}$ the last one is down from $150 \mathrm{rad} / \mathrm{s}$ to $50 \mathrm{rad} / \mathrm{s}$ at $2.5 \mathrm{~s}$. Figure $4 \mathrm{a}$ shows that, the speed response is good, which presents a small responses time $0.3 \mathrm{~s}$. The induction machine is loaded by 10 N.m at $0.5 \mathrm{~s}$ as showing in Figure $4 \mathrm{~b}$. We can see in Figure $4 \mathrm{~b}$ that the torque has a good response. Figure $4 \mathrm{~b}$, Figure $4 \mathrm{c}$ and Figure $4 \mathrm{~d}$ show that the currents, voltages and torque respect the physical limits of the induction machine. Figure $4 \mathrm{e}$ shows that the decoupling between the torque and the flux is correct, and that the flux and speed estimation error are very low as showing in Figure $4 \mathrm{f}$ and Figure $4 \mathrm{~g}$. Figure $4 \mathrm{~h}$ shows the evolution of the ratio of the estimated stator resistance and the nominal value of the stator resistance, in this simulation we suppose that stator resistance doesn't varies. We can see that the estimation response is good.

The Figure 5 shows us the simulation responses of the asynchronous machine commanded for $100 \mathrm{rad} / \mathrm{s}$ of speed Figure $5 \mathrm{a}$, and for a load torque steps Figure $5 \mathrm{~b}$, the first step is given at $0.5 \mathrm{~s}$ from 0 to $10 \mathrm{~N} . \mathrm{m}$, the second one is from $10 \mathrm{~N} . \mathrm{m}$ to $20 \mathrm{~N} . \mathrm{m}$ at $1.5 \mathrm{~s}$, and the third one is down from 20 N.m to 5 N.m at $2.5 \mathrm{~s}$. We can observe in Figure $5 \mathrm{~b}$ that the motor torque follows the load torque. Also, we notice that stator currents and stator voltage still under maximum limits values of the induction machine as showing in Figure $5 \mathrm{c}$ and Figure $5 \mathrm{~d}$. Simulation results show that the speed and the flux in Figure 5e are not influenced by torque variation. We can see in Figure 5f, Figure 5g and Figure 5h that the estimation errors, of speed, rotor flux and stator resistance are very weak.

The Figure 6 shows the simulation responses of the system commanded for $100 \mathrm{rad} / \mathrm{s}$ in speed (Figure 6.a) and 10N.m in load torque (Figure 6.b) with a filtered steps variation of $70 \%$ of the nominal stator resistance $\left(R_{s n}=2.2 \Omega\right)$ coming at $1.5 \mathrm{~s}$ and another one given at $2.5 \mathrm{~s}$ from $70 \%$ to $30 \%$ of the nominal stator resistance. We can see that the control present a stable and acceptable response. We notice that the responses of speed (Figure 6.a), couple (Figure 6.b) and flux (Figure 6.c) shows that the influence of this variation is null, and that estimation errors of speed (Figure 6.d), flux (Figure 6.e)and stator resistance remains null (Figure 6.f).

The parameters of Induction Machine used in simulation are given below:

Table 1. The parameters of the Induction Machine:

\begin{tabular}{lccc}
\hline \hline \multicolumn{1}{c}{ Parameters } & & Values & Units \\
\hline Rated power & $\mathrm{P}$ & 3 & $\mathrm{kw}$ \\
Voltage & $\mathrm{U}$ & 380 & $\mathrm{~V}$ \\
Rated current & $\mathrm{I}$ & 7.3 & $\mathrm{~A}$ \\
Rated speed & $\mathrm{n}$ & 1440 & $\mathrm{rpm}$ \\
Stator Resistance & $\mathrm{Rs}$ & 2.2 & $\Omega$ \\
Rotor Resistance & $\mathrm{Rr}$ & 2.68 & $\Omega$ \\
Mutual Inductance & $\mathrm{Lm}$ & 0.217 & $\mathrm{H}$ \\
Stator Inductance & $\mathrm{Ls}$ & 0.229 & $\mathrm{H}$ \\
Rotor Inductance & $\mathrm{Lr}$ & 0.229 & $\mathrm{H}$ \\
Motor load inertia & $\mathrm{J}$ & 0.047 & $\mathrm{~kg} . \mathrm{m}^{2}$ \\
Viscous friction coefficient & $\mathrm{f}$ & 0.004 & $\mathrm{N.s} / \mathrm{rad}$ \\
Number of pole pairs & $\mathrm{p}$ & 2 & \\
\hline \hline
\end{tabular}



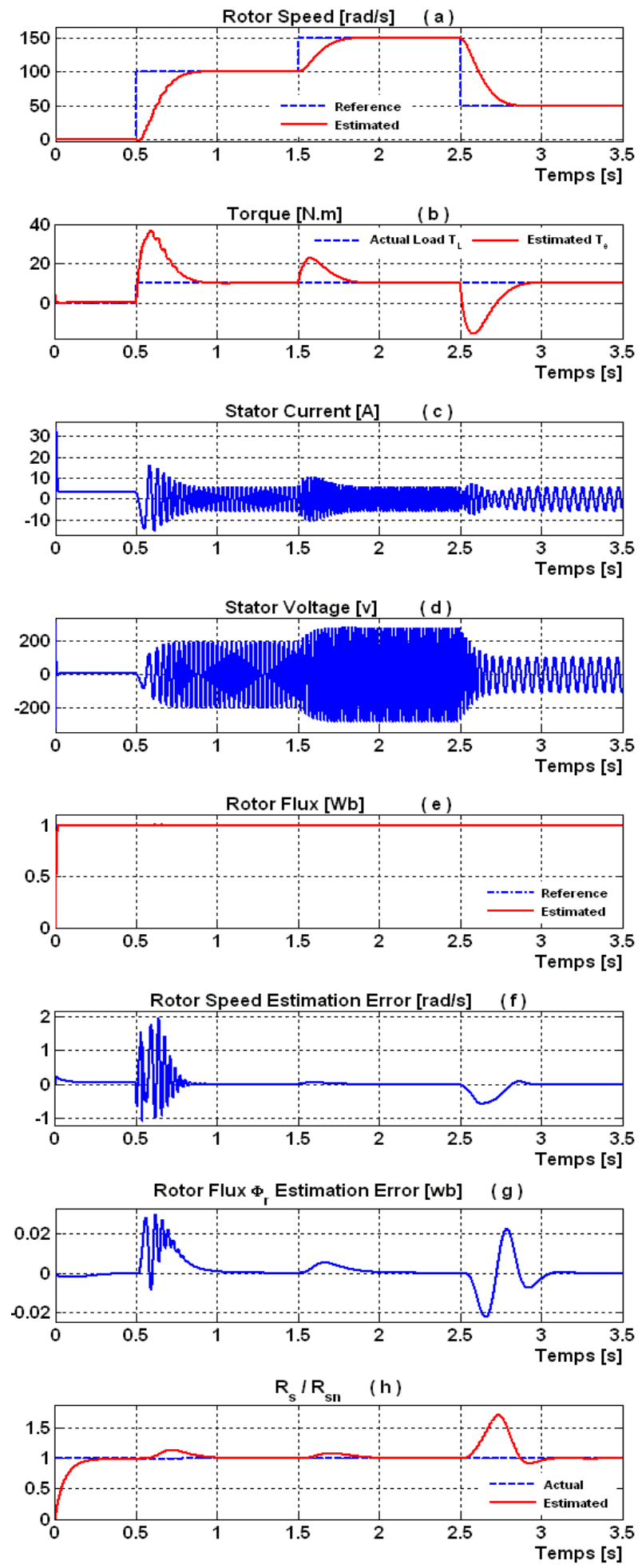

Figure 4. Simulation responses for steps of reference speed for 10N.m in load torque. 
Mohamed Moutchou, et al.
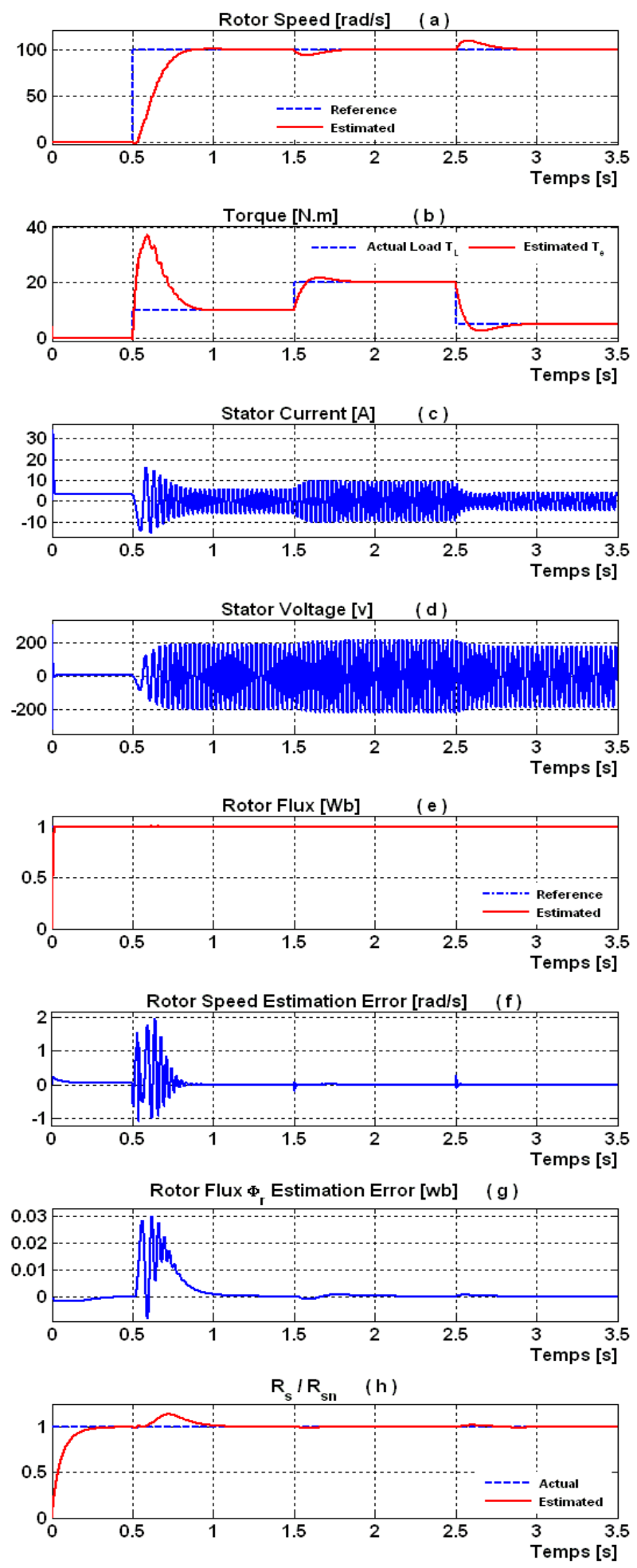

Figure 5. Simulation responses for steps of load torque with $100 \mathrm{rad} / \mathrm{s}$ in reference speed. 

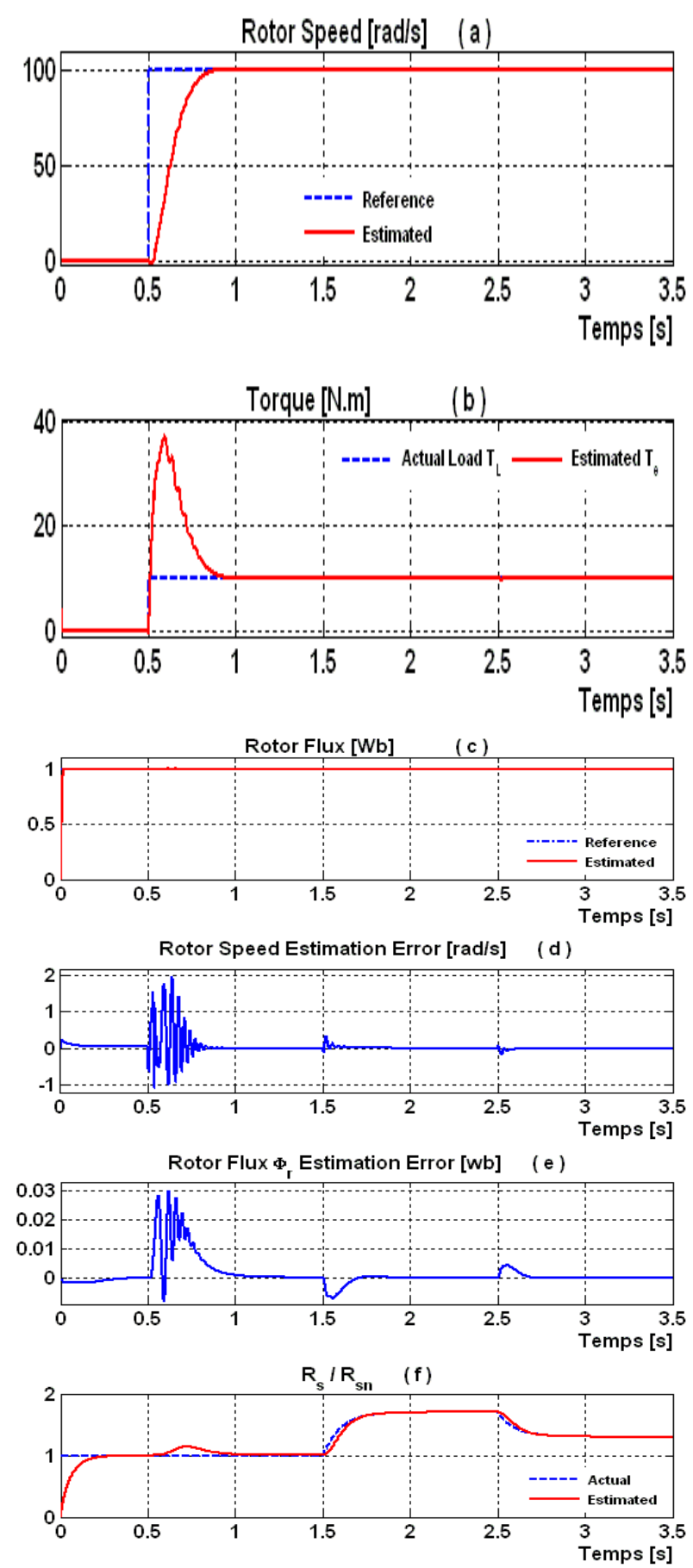

Figure 6. Simulation responses for stator resistance variation with $100 \mathrm{rad} / \mathrm{s}$ in reference speed and $10 \mathrm{~N} . \mathrm{m}$ in load torque.

\section{Conclusion}

In this article, we made a study of the sensorless speed control of the induction machine using exact input-output linearization control technique, associated to a special MRAS observer that provides simultaneous estimations of rotor speed and stator resistance, governed 
by an adaptation law according to the stator current and rotor flux estimated errors. The estimation of flux component was obtained by using a sliding mode observer. The simulation results showed that this control and observation techniques combination presents of maid good performances and allows a complete decoupling between the flux and the torque. These performances are obtained with stator current, stator voltage and torque that respect the physical limits of the induction machine.

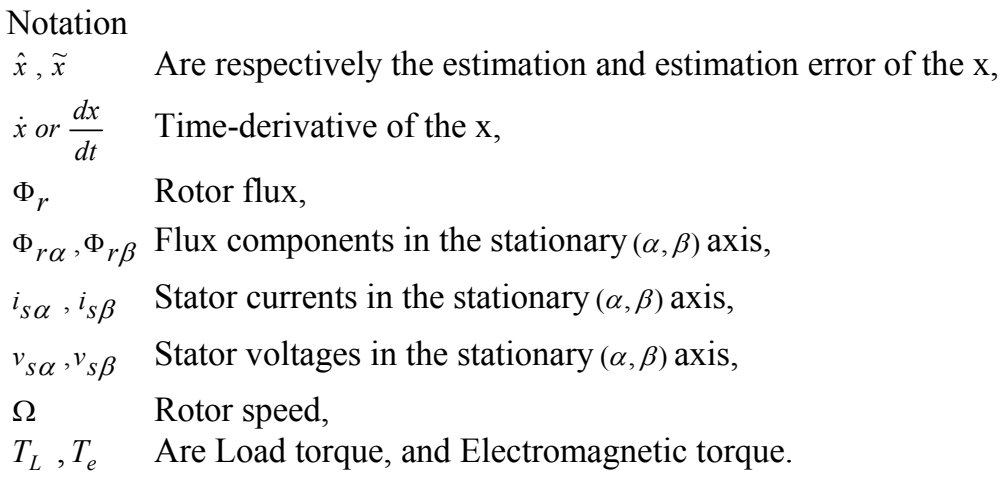

\section{References}

[1] F. Blaschke, "The principle of field orientation applied to the transvector closed-loop control system for rotating field machines," Siemens Rev., Vol. 34, pp. 217-220, 1972.

[2] Z. Krzeminski, "Nonlinear control of the induction motor," in Proc. 10th IFAC World Congress, Munich, Germany, 1987, pp.349-354.

[3] D. Casadei, G. Grandi, G. Serra, "Rotor Flux Oriented Torque-Control of Induction Machines Based on Stator Flux Vector Control", Proceedings of the EPE Conference, Brighton, Vol. 5, pp. 67-72, 1993.

[4] A. Abbou, H. Mahmoudi, "Implementation of a Sensorless speed control Of Induction Motor using RFOC strategy", International Review of Electrical Engineering, Vol.3, pp.730-737, 2008.

[5] V.I. Utkin, "Sliding mode control design principles and applications to electric drives", IEEE Transactions On Industrial Electronics, Vol. 40, pp. 23-36, 1993.

[6] A. Sabanovic, D.B Izosimov, "Application of sliding modes to induction motor control", IEEE Transactions on Industry Applications, IA-17, pp. 344-348, 1981.

[7] C. Edwards, S. Spurgeon, "Robust output tracking using a sliding mode Controller observer scheme ", Int. Jour. of Cont., Vol. 64, pp. 967-983, 1996.

[8] G. Bartolini, A. Ferrara, E. Usai, "Chattering Avoidance by Second Order Sliding Mode control", IEEE transactions on Automatic Control, Vol. 43, pp. 241-246, 1998.

[9] R. Ortega, G. Espinoza, "Torque regulation of induction motor", Automatica (Journal of IFAC), Vol. 29, pp. 621-633, 1993.

[10] R. Ortega, P.J. Nicklasson, G.E. Péres, "On speed control of induction motors", Automatica, Vol. 32, pp. 455-460, 1996.

[11] R.A. Freeman, P.V. Kokotovic, "Backstepping design of robust controllers for a class of nonlinear systems", Proceedings of IFAC Nonlinear Control Systems Design Symposium, Bordeaux France, pp. 307-312, 1992.

[12] F. Ikhouane, M. Krstic, "Adaptive backstepping with parameter projection: robustness and asymptotic performance" Automatica, Vol. 34, pp. 429-435, 1998.

[13] H. Tan, J.Chang, "Adaptive backstepping control of induction motor with uncertainties", Proceedings of the American control conference, San Diego, California, Vol. 1, pp. 1-5, 1999. 
[14] J. Zhou, C. Wen, Adaptive Backstepping Control of Uncertain Systems: Nonsmooth Nonlinearities, Interactions or Time-Variations, Berlin Heidelberg Springer-Verlag 2008.

[15] M. Moutchou, A. Abbou, H. Mahmoudi," Sensorless Speed Backstepping Control of Induction Machine, Based On Speed MRAS Observer" International Conference on Multimedia Computing and Systems (ICMCS'12) Tangier, Morocco 2012.

[16] M. Bodson, J. Chiasson, R. Novotnak, "High-Performance Induction Motor Control Via Input-Output Linearization", IEEE Control Syst. Mag., Vol. 14, pp. 25-33, 1994.

[17] R. Marino, S. Peresada, and P. Valigi, "Adaptive partial feedback linearizing of induction motors," Proc. 29th Conf Decision and Control, Honolulu, HI, Dec. 1990.

[18] R. Marino, P. Tomei, Nonlinear Control Design, Geometric, Adaptive and Robust, Prentice-Hall, New York, 1995.

[19] A. Isidori, Nonlinear Control Systems, Third Edition, Springer-Verlag London, 1995.

[20] A. De Luca, G. Ulivi, "Design of an Exact Nonlinear Controller for Induction Motors", IEEE Transactions on Automatic Control, vol. 34, no. 12, pp. 1304-1307, 1989.

[21] J. Chiason, "A new approach to dynamic feedback linearization control of an induction motor," IEEE Transactions on Automatic Control, vol.43, N3, pp.391-397, 1997.

[22] M. Bodson, J. Chiasson, R. Novotnak, "High-Performance Induction Motor Control Via Input-Output Linearization” IEEE Control Syst. Mag., vol. 14, no. 4, pp. 25-33, 1994.

[23] M. Moutchou, A. Abbou , H. Mahmoudi, M. Akherraz, "Sensorless Input-Output Linearization Speed Control of Induction Machine", The international workshop on Information Technologies and Communication Wotic'11, ID.123, 2011.

[24] L. Zhen, L. Xu, "Sensorless field orientation control of induction machines based on mutual MRAS scheme", IEEE Transactions on Industrial Electronics, Vol. 45, pp. 824831, 1998.

[25] M. Rashed, A.F. Stronach, "A stable back-EMF MRAS-based sensorless low speed induction motor drive insensitive to stator resistance variation," IEE Proceedings Electric Power Applications, Vol. 151, pp. 685-693, 2004.

[26] Y.A. Kwon, D.W. Jin, "A novel MRAS based speed sensorless control of induction motor," in Proc. the 25th Annual Conference of the IEEE Industrial Electronics Society, Vol. 2, pp. 933-938, 1999.

[27] C. Schauder, "Adaptive speed identification for vector control of induction motors without rotational transducers," IEEE Transactions on Industry Applications, Vol. 28, pp. 1054-1061, 1992.

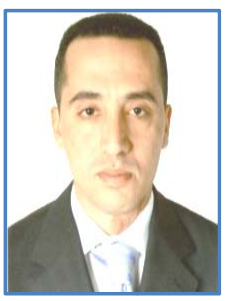

Mohamed Moutchou was born in KHINIFRA, Morocoo, in 1974. He received the B.S degree in Electronics from ENSET Mohammedia, Morocco in 1998 and Aggregation in electrical engineering from ENSET Rabat, Morocco in 2006, and received the M.S. degree in industrial electronics from Mohammadia School's of Engineers, Morocco, in 2010.

Since 1998 he has taught in various academic institutions in Morocoo. Since 2010 as $\mathrm{PhD}$ student, his current research interests are design and implementation of non-linear controls of electrical machine. m.moutchou@yahoo.fr

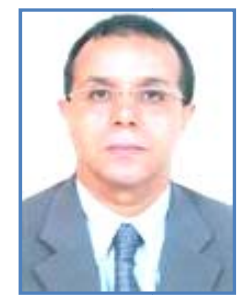

Hassan Mahmoudi was born in Meknes, Morocco, on January 4, 1959. He received the B.S degree in electrical engineering from Mohammadia School's of engineers, Rabat, Morocco, in 1982, and the Ph.D. degree in power electronic from Montefiore Institute of electrical engineering, Luik, Belgium, in 1990. 
Mohamed Moutchou, et al.

He was an Assistant Professor of physics, at the Faculty of sciences, Meknes, Morocco, from 1982 to 1990. Since 1992, he has been a Professor at the Mohammadia School's of engineers, Rabat, Morocco, and he was the Head of Electric Engineering Department during four years (1999, 2000, 2006 and 2007).

His research interests include static converters, electrical motor drives, active power filters and the compatibility electromagnetic.

mahmoudi@emi.ac.ma 